\title{
Simulation of Bennet and Brassard 84 protocol with Eve's attacks
}

\begin{abstract}
A simulation of BB84 protocol of QKD using photonic simulator OptiSystem is demonstrated. The simulation is divided into two categories which are without security attacks scenario and with several attacks scenario that consists of Intercept and Resend and sent new qubits. The simulation results meet the theory of BB84. This project is able to provide better understanding about the quantum key distribution process especially in BB84 protocol.
\end{abstract}

Keyword: BB84 protocol; Cryptography; Optisystem; Quantum cryptography; Quantum key distribution 